\title{
Aplicación de la Ecuación del Balance Térmico para determinar el confort higrotérmico en la vivienda unifamiliar en el cantón Girón.
}

\section{Application of the Thermal Balance Equation to determine hygrothermal comfort in a single-family house in canton Giron.}

Cristián Raúl Guzmán Clavijo. ${ }^{1}$ \& Doris Alexandra Alvear Calle. ${ }^{2}$

\begin{abstract}
.
DOI: https://doi.org/10.33262/cienciadigital.v5i2.1722

The single-family houses in Girón, a canton located in the south of the province of Azuay, reveals the lack of hygrothermal performance studies in the design process that achieve successful internal thermal comfort conditions for the users; scenario that falls into poor indoor environmental quality of life. Through the thermal equilibrium, applying the heat balance equation, the research aims to determine the level of comfort or discomfort that the user experiences in the different the single-family house inner spaces. A qualitative research, starting at the exploratory level, with non-probabilistic sampling and the application of surveys, allows to obtain Girón population data. Heat balance calculations reveals if whether people are in comfort or in discomfort. The results exhibit that there are several factors which ward off the house users from being within the comfort zone; accordingly, it evidences the importance of the thermal balance analysis, which opens a

\footnotetext{
${ }^{1}$ Universidad Católica de Cuenca, Facultad de Arquitectura, Cuenca, Ecuador. cguzman23@ucacue.est.edu.ec

${ }^{2}$ Universidad Católica de Cuenca, Facultad de Arquitectura. Cuenca, Ecuador. doris.alvear@ucacue.edu.ec
} 
wide range of possible recommendations that will allow reaching the expected levels comfort.

Keywords: hygrothermal comfort, convection, conduction, metabolism, radiation, evaporation.

\section{Resumen.}

En el cantón Girón ubicado al sur de la provincia del Azuay, se evidencia que las viviendas unifamiliares carecen de diagnósticos higrotérmicos en el proceso de diseño que garanticen las condiciones de confort del usuario, ocasionando malestar en las condiciones de habitabilidad al interior de la vivienda. Mediante la aplicación de la ecuación del balance térmico se pretende determinar el grado de confort o disconfort que experimenta el usuario en los diferentes espacios al interior de la vivienda unifamiliar. A través de una investigación cualitativa que parte del nivel exploratorio, con el apoyo del muestreo no probabilístico y la aplicación de encuestas, se obtiene información de la población gironense que bajo el análisis del balance térmico determina si se encuentra en confort o en disconfort. Los resultados obtenidos exponen que son diversos lo factores que impiden que los habitantes se encuentren dentro de la zona de confort, evidenciando como consecuencia la importancia del análisis del balance térmico, el mismo que abre un abanico de posibilidades para plantear recomendaciones que permitan alcanzar los niveles de confort anhelados.

Palabras claves: confort higrotérmico, convección, conducción, metabolismo, radiación, evaporación.

\section{Introducción.}

La arquitectura, ese el arte de proyectar y construir, sin importar los estilos arquitectónicos, tiene la finalidad de brindar satisfacción y las condiciones adecuadas para los habitantes y sus relaciones con el entorno, es por ello que debe palpar el territorio en el cual se construirá ya sea un edificio, hospital, vivienda, etc. y relacionarlo con los factores climáticos con los cuales tendrá que trabajar para conseguir el confort deseado para el hábitat de las personas; sin duda de los diferentes espacios que se pueden construir, la vivienda es el que resulta más factible de cumplir las expectativas y exigencias de confort (Cancino y Cortés, 2015).

Existen diferentes factores que ayudan a determinar el confort como son: los factores internos y factores externos, en lo que respecta a factores externos podemos decir que se refiere al grado de arropamiento, tipo y color de vestimenta, factores ambientales como temperatura de aire, temperatura radiante, humedad del aire, radiación, velocidad del viento, niveles lumínicos, niveles acústicos, calidad del aire, olores, ruidos, elementos visuales, etc. (Murillo, 2011) considerando la diversidad a la que se refiere se puede subdividir en dos tipos: los factores físicos o climáticos en los que se encuentra la temperatura del aire, radiación, humedad y el movimiento del aire; y los factores 
individuales que abarcan la vestimenta, aclimatación, sexo, edad, forma del cuerpo, grasa subcutánea, alimentos y bebidas, color de la piel y estado de salud.

En cuanto a los factores internos que ayudan a determinar el confort tenemos: la raza, sexo, edad, características físicas y biológicas, salud física o mental, estado anímico, grado de actividad metabólica, experiencias y asociación de ideas, entre otros (Murillo, 2011). De acuerdo a la percepción sensorial tenemos diferentes tipos de confort: higrotérmico, lumínico, acústico, olfativo y psicológico. De los cuales nos centraremos en el primero el confort higrotérmico.

Hablar de confort higrotérmico en muchos casos quizás considerarían irrelevante o innecesario, pero cuando de construcciones se trata este tema lleva sin duda una inclinación bastante significativa, pues consideremos que los seres humanos queremos vivir en una edificación tranquila en la que, no solo estemos aislados de ruidos externos sino también deseamos ese lugar en el que no necesitemos recubrirnos con exagerada vestimenta porque hay un día lluvioso y temperaturas bajas en el ambiente, o por el contrario abrir ventanas o poner aire acondicionado porque el día está soleado y con temperaturas elevadas. Según Murillo (2011), el confort higro-térmico se puede definir como la ausencia de malestar térmico, y para que exista el mismo, los mecanismos termorreguladores del cuerpo no deben intervenir, éste confort se alcanza con una temperatura entre $21^{\circ} \mathrm{C} \mathrm{y} 25^{\circ} \mathrm{C}$, y con una humedad relativa entre el $20 \%$ y $75 \%$.

El equilibrio térmico del cuerpo humano puede expresarse mediante una ecuación denominada balance térmico del cuerpo humano, en la que se representan los factores de ganancia (termogénesis) y pérdida (termólisis).

Tabla 1. Factores de ganancia

\begin{tabular}{ll}
\hline \multicolumn{1}{c}{ FACTORES } & \multicolumn{1}{c}{ DESCRIPCIÓN } \\
\hline Metabolismo & $\begin{array}{l}\text { Del proceso basal, proceso digestivo, actividad y tensión muscular. } \\
\text { Rel sol, directa y reflejada, de radiadores incandescentes y de objetos } \\
\text { Radiación }\end{array}$ \\
$\begin{array}{l}\text { Conducción } \\
\text { Convección no incandescentes. }\end{array}$ & Por contacto con cuerpos calientes. \\
\hline
\end{tabular}

Fuente: Murillo,2011.

Elaborado: Cristian Guzmán

Tabla 2. Factores de pérdida

\begin{tabular}{ll}
\hline \multicolumn{1}{c}{ FACTORES } & \multicolumn{1}{c}{ DESCRIPCIÓN } \\
\hline Radiación & Al cielo y superficies frías. \\
Conducción & Por contacto con cuerpos fríos. \\
Convección & Al aire con temperatura menor que la piel. \\
Evaporación & Por respiración y transpiración. \\
\hline
\end{tabular}

Fuente: Murillo,2011.

Elaborado: Cristian Guzmán 
Existirá éste determinado equilibrio térmico cuando el ambiente esté térmicamente neutro, esto quiere decir cuando la termogénesis se equilibra con la termólisis, no se almacena calor y la temperatura corporal se equilibra (Murillo, 2011), es decir:

$$
\mathrm{M}+/-\mathbf{R}+/-\mathbf{C d}+/-\mathbf{C v}-\mathbf{E}=\mathbf{0}
$$

Ecuación 1.

Donde:

$\mathrm{M}=$ metabolismo

$\mathrm{R}=$ radiación (porcentaje en relación al metabolismo)

$\mathrm{Cd}=$ conducción

$\mathrm{Cv}=$ convección

E=evaporación

Si la suma es mayor a 0 quiere decir que el cuerpo se está calentando y se producirán regulaciones vasomotoras, por otro lado, si la suma es menor a 0 quiere decir que el cuerpo se está enfriando. El ser humano tiene una temperatura interna propia e independiente del ambiente del medio externo, regularmente esta temperatura es de $36.5^{\circ} \mathrm{C}$ a $37^{\circ} \mathrm{C}$, sin embargo, el ser humano puede soportar temperaturas internas entre $25^{\circ} \mathrm{C}$ a $42^{0} \mathrm{C}$, pero por períodos cortos de tiempo. El cuerpo humano puede ser considerado como una máquina térmica que necesita una cierta cantidad de calor para que pueda funcionar, la principal fuente de calor para el organismo es la producción del calor metabólico (M), el cuerpo de las personas produce calor ya sea estando en reposo o en movimiento, una parte de éste se transmite al ambiente en un porcentaje estimado de un $80 \%$ y tan solo un $20 \%$ es utilizada (Murillo, 2011).

Es importante indicar que para desarrollar la ecuación de balance térmico se requiere primero obtener el valor de cada uno de los componentes de la ecuación a través de sub ecuaciones de; Metabolismo, Radiación, Conducción, Convección y Evaporación.

\section{Metabolismo}

Para conocer el valor de metabolismo (M) se aplica la siguiente ecuación elaborada por Harris Benedict la cual se aplica para determinar la tasa metabólica:

Tabla 3. Ecuación de Harris Benedict

\begin{tabular}{ll}
\hline HOMBRES & $\begin{array}{l}\text { TMB }=(10 \times \text { peso en } \mathrm{kg})+(6,25 \times \text { altura en } \mathrm{cm})-(5 \times \\
\text { edad en años })+5\end{array}$ \\
\hline MUJERES & $\begin{array}{l}\text { TMB }=(10 \times \text { peso en } \mathrm{kg})+(6,25 \times \text { altura en } \mathrm{cm})-(5 \times \\
\text { edad en años })-161\end{array}$
\end{tabular}

Fuente: Murillo,2011.

Elaborado: Cristian Guzmán

Para conocer el metabolismo de acorde a las diferentes actividades, se emplea la siguiente tabla, la cual está calculada para una superficie corporal de $1.72 \mathrm{~m}^{2}$. 
ISSN: 2602-8085

www.cienciadigital.org

Vol. 5, N², p. 149-164, Abril-Junio, 2021

Tabla 4. Tabla de metabolismo de las diferentes actividades.

\begin{tabular}{lrrr}
\hline ACTIVIDAD & W/m2 & Met & W/persona \\
\hline Dormir & 40 & 0,70 & 69 \\
Estar acostado & 45 & 0,80 & 77 \\
Sentado con movimiento moderado & 60 & 1,00 & 103 \\
Sentado con actividad ligera & 64 & 1,10 & 110 \\
De pie sin movimiento & 70 & 1,20 & 120 \\
De pie con actividad ligera & 78 & 1,30 & 134 \\
De pie con levantamiento y transporte moderados & 93 & 1,60 & 160 \\
Trabajo manual ligero & 100 & 1,70 & 172 \\
Caminar en horizontal (2km) & 110 & 1,90 & 189 \\
Bailar (actividad social) & 111 & 1,90 & 191 \\
Construcción ligera & 125 & 2,20 & 215 \\
Trabajo manual moderado & 139 & 2,40 & 239 \\
Lavar platos & 145 & 2,50 & 249 \\
Limpieza doméstica & 150 & 2,60 & 258 \\
Ejercicio moderado & 167 & 2,90 & 287 \\
Lavar a mano, planchar & 170 & 2,90 & 292 \\
Construcción moderada & 180 & 3,10 & 310 \\
Caminar en horizontal (5Km) & 200 & 3,40 & 344 \\
Trabajo manual pesado & 235 & 4,10 & 404 \\
Ejercicio intenso & 250 & 4,30 & 430 \\
Construcción pesada & 275 & 4,70 & 473 \\
Ejercicio o trabajo muy intensos & 450 & 7,80 & 774 \\
Correr (15 Km) & 550 & 9,50 & 946 \\
\hline Fuente: Murllo
\end{tabular}

Fuente: Murillo,2011.

Elaborado: Cristian Guzmán

En el caso de que se desee calcular la superficie corporal de un individuo en etapa adulta se utiliza la siguiente ecuación de Mosteller:

$$
\mathrm{X}=\sqrt{(\text { peso }} * \text { altura }) / 3600
$$

Ecuación 2.

Mientras que para niños se aplica la ecuación de Haycock:

$\mathrm{X}=0,024265 *$ peso $(\mathrm{kg})^{0,5378 *}$ altura $(\mathrm{cm})^{0,3964}$

Ecuación 2.1.

Radiación.

Para conocer el valor R (radiación) se aplica la siguiente ecuación:

$$
\mathbf{R}=\mathrm{e}^{*} \boldsymbol{d}^{*} \mathbf{A} * \Delta \mathrm{T}
$$

Ecuación 3.

En la que: 
$\mathrm{R}=$ flujo de calor por radiación $(\mathrm{W})$

e= emisividad-absorbencia de 0.7 a 0.9 , dependiendo de la pigmentación, para ello se puede basar en la siguiente tabla:

Tabla 5. Tipos de piel

\begin{tabular}{|c|c|c|c|}
\hline TIPO & NOMBRE & CARACTERISTICAS & (e) ESTIMADO \\
\hline TIPO I & Céltica & $\begin{array}{l}\text { Piel muy pálida, cabello } \\
\text { rojizo, muchas pecas }\end{array}$ & 0,65 \\
\hline TIPO II & Pálido & $\begin{array}{l}\text { Piel pálida, cabello rubio, } \\
\text { algunas pecas }\end{array}$ & 0,70 \\
\hline TIPO III & Caucásica & $\begin{array}{l}\text { Piel caucásica, cabello } \\
\text { castaño, sin pecas }\end{array}$ & 0,75 \\
\hline TIPO IV & Mediterránea & $\begin{array}{l}\text { Piel marrón, cabello y ojos } \\
\text { oscuros }\end{array}$ & 0,80 \\
\hline TIPO V & $\begin{array}{c}\text { Indio } \\
\text { americano }\end{array}$ & $\begin{array}{c}\text { Piel marrón oscuro, cabello } \\
\text { oscuro }\end{array}$ & 0,85 \\
\hline TIPO VI & Negra & Piel y cabello negro & 0,90 \\
\hline
\end{tabular}

Fuente: Murillo,2011.

Elaborado: Cristian Guzmán

A: porción de la superficie corporal de la persona en $\mathrm{m}^{2}$.

ð.5.6703* $10^{-8}$ watt $/ \mathrm{m}^{2} \mathrm{k}^{4}$

AT: diferencia de temperatura $\left(\mathrm{k}^{4}\right.$ grados kelvin $)$

AT: $\mathrm{tn}^{4}-\mathrm{ta}^{4}$

Tn: temperatura neutra $\left(\mathrm{k}^{4}\right.$ grados kelvin), que hace referencia a la temperatura en la que el cuerpo tiene el menor esfuerzo para mantener su equilibrio térmico con el medio circundante, y para calcularlo se aplica la siguiente fórmula de Szokolay.

$$
\begin{aligned}
& \operatorname{Tn}= 17,6+0,31 \mathrm{Tm} \\
& \mathrm{Zc}=\operatorname{Tn}+/-2,5^{\circ} \mathrm{C}
\end{aligned}
$$

Ecuación 4.

Ecuación 5.

Tn: Temperatura neutra $\left({ }^{0} \mathrm{C}\right)$

Tm: temperatura media $\left({ }^{0} \mathrm{C}\right)$

Zc: zona de confort

\section{Convección.}

Para conocer el valor Cv (convección) tenemos la ecuación:

$$
\mathrm{Cv}=\mathrm{hc}^{*} \mathrm{~A} * \Delta \mathrm{T}
$$

Ecuación 6.

Cv: flujo de energía calorífica

hc: coeficiente de convección $\left(\mathrm{W} / \mathrm{m}^{2} \mathrm{c}^{0}\right)$, para superficies expuestas a vientos exteriores se emplea la siguiente ecuación, en la que v=velocidad del viento $(\mathrm{m} / \mathrm{sg})$. 
A: porcentaje de superficie corporal $\left(\mathrm{m}^{2}\right)$

$\Delta \mathrm{T}$ : diferencia de temperatura $\left({ }^{0} \mathrm{C}\right)$

$\Delta \mathrm{T}$ : diferencia de temperatura $\left(\mathrm{k}^{4}\right.$ grados kelvin)

$\Delta \mathrm{T}: \mathrm{tn}^{4}-\mathrm{ta}^{4}$

\section{Conducción.}

Para conocer el valor conducción $(\mathrm{Cd})$ tenemos que conocer acerca de la misma, pues la Cd no es más que la transmisión del calor entre la piel y los elementos en contacto, es decir con la ropa, ya que ésta se opone al paso del calor por lo que el hombre se protege del frio arropándose y del calor desnudándose. La integración de la vestimenta en el proceso de intercambio se realiza mediante la definición de una unidad de aislamiento

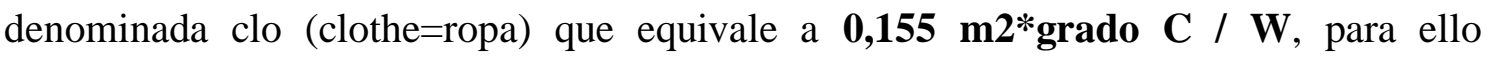
empleamos la tabla con el valor clo.

Tabla 6. Valores clo de acuerdo a combinaciones de vestuario.

\begin{tabular}{lrr}
\hline \multicolumn{1}{c}{ NIVEL DE VESTUARIO } & Clo & M2 * grado C / W \\
\hline Cuerpo desnudo & 0 & 0 \\
Bikini similar & 0,05 & 0,008 \\
$\begin{array}{l}\text { Ropa tropical: interior ligero + camisa corta + pantalón } \\
\text { corto }\end{array}$ & 0,20 & 0,031 \\
$\begin{array}{l}\text { Ropa ligera: interior ligera + camisa corta + pantalón } \\
\text { ligero }\end{array}$ & 0,50 & 0,078 \\
Traje tropical & 0,80 & 0,124 \\
Traje formal de negocios incluido chaleco & 1,00 & 0,155 \\
Traje con abrigo de lana y ropa interior larga & 1,60 & 0,248 \\
Ropa de esquimales & 3,50 & 0,546
\end{tabular}

Fuente: Murillo,2011.

Elaborado: Cristian Guzmán

Una vez que entendido el valor clo se procede a la ecuación para obtener el valor de la conductividad:

Donde:

Cd: flujo de energía calorífica por conducción (W)

A: área expuesta al flujo de calor $\left(\mathrm{m}^{2}\right)$ 
$\mathrm{Ct}$ : conductacia total $\left(\mathrm{W} / \mathrm{m}^{2}{ }^{\circ} \mathrm{C}\right)$

$\mathrm{Ct}: \mathrm{Cc}-\mathrm{Cm}$

$\mathrm{Cc}$ : conductancia corporal $(\mathrm{k} / \mathrm{b})$

$\mathrm{k}$ : conductividad del material, piel $\left(0,34 \mathrm{~W} / \mathrm{m}^{\circ} \mathrm{C}\right)$

b: espesor del material, piel $(0,001 \mathrm{~m})$

$\mathrm{Cm}$ : conductancia del material $\left(\mathrm{W} / \mathrm{m}^{2}{ }^{\circ} \mathrm{C}\right)$

$\Delta \mathrm{T}$ : diferencia de temperaturas $\left({ }^{\circ} \mathrm{C}\right)$

\section{Evaporación.}

Para conocer el valor E (evaporación) tenemos la ecuación:

$$
\mathrm{Qe}=666,66 * \mathrm{e}^{*} \mathrm{v}
$$

\section{Ecuación 9.}

En el que:

Qe: pérdida total por vaporación en watts (W)

ev: tasa de evaporación en $\mathrm{kg} / \mathrm{h}$, este valor es una constante y es igual a $1,5 \mathrm{~kg} / \mathrm{h}$, pero podría variar según las características de las personas.

Cancino y Cortés (2015) realizaron una investigación cuyo objetivo principal era analizar cuánto y cómo inciden los mejoramientos técnicos de la MART en el conforthigrotérmico el hábitat residencial para lo cual seleccionaron tres casos de estudio, encuestas de percepción a los habitantes y comparación de resultados, todo ello para demostrar la importancia del confort higrotérmico $(\mathrm{CH})$ en una vivienda y como un mejoramiento técnico puede incidir en la percepción de quienes habitan. Diulio et al (2012), consideran la importancia del estudio e implementación de técnicas de análisis y de aplicación de métodos para mejorar el confort higrotérmico en las viviendas para ello utilizan microadquisidores de datos HOBO. Otros autores también hablan con respecto al confort higro-térmico mencionando a las variables de temperatura, humedad y ventilación de los espacios habitados y su relación directa con del diseño y reglamentación técnica, con las características de la vivienda, con el clima del entorno y las prácticas sociales de los habitantes (Lisandro, 2015), lo que da a conocer la necesidad del estudio y aplicación de técnicas adecuadas para la construcción de una vivienda.

Son pocos los estudios realizados sobre $\mathrm{CH}$ y por ende son muchas las incertidumbres que se presentan, lo que genera la necesidad de realizar un estudio más profundo sobre ésta temática que permita dar respuesta a las necesidades de las personas, pues no es una novedad escuchar a los propietarios de viviendas decir que: su casa es fría, que se sofocan en épocas de verano, que la humedad impide que puedan descansar en las noches, y otras frases más; sin duda la falta de criterio técnico en el momento de construir en el que personas no especializadas en la rama de la arquitectura meten su mano para edificar, y no sólo eso también la falta de recursos económicos o el inmediatismo de los propietarios hacen que éste apartado crucial para su homeostasis, sea desplazado, dejándolo en 
segundo plano y acarreando posteriormente con las consecuencias dentro de las cuales la principal es la incomodidad.

\section{Metodologia.}

La investigación parte de un objetivo principal el cual consiste en aplicar la ecuación del balance térmico, a la población del cantón Girón para determinar el nivel de confort higrotérmico, éste proceso se lleva a cabo a través del uso de una investigación cualitativa, que parte del nivel exploratoria, ya que es un campo nuevo de estudio que deja una puerta abierta a nuevas y futuras investigaciones; éste tipo de investigación tiene dos metas principales la comprensión y descripción de los fenómenos en un contexto determinado, en éste caso las características físicas y comportamentales de la persona y las especificaciones técnicas de los interiores de la vivienda; el tipo de muestreo a utilizar, es el muestreo no probabilístico, considerando que las familias a participar fueron voluntarias; el diseño con el cual se enfoca éste proceso investigativo es la investigaciónacción, ya que está encaminada en un futuro a poder resolver el problema de confort en la vivienda partiendo de una base teórica.

\section{Población y muestra}

Girón es un cantón ubicado al sur de la provincia de Azuay, de acuerdo al Instituto Nacional de Estadísticas y Censos (INEC) existen 3481 familias integradas cada una por 3.59 integrantes, con la finalidad de obtener información del promedio de habitantes del cantón para el cálculo del balance térmico, se empleará la técnica de encuestas, para determinar número de familias que deben ser encuestadas se aplica la ecuación de muestreo estratificado:

$$
n=\frac{k^{2} * P * Q * N}{e^{2}(N-1)+k^{2} * P * Q}
$$

Donde:

N:es el tamaño de la población o universo (número total de familias a encuestar)

$\mathrm{k}$ : constante que dependerá del nivel de confianza que se asigne, el nivel de confianza indica la probabilidad de que los resultados de nuestra investigación sean ciertos: en este caso se utiliza un $90 \%$ de nivel de confianza, por ende $\mathrm{K}=1.65$.

e: error muestral deseado, es decir, viene a ser la diferencia que puede haber entre el resultado que obtenemos, preguntando a una muestra de la población y el que obtendríamos si preguntásemos al total de la población.

p: es la proporción de individuos que poseen en la población la característica del estudio, éste dato por lo general tiende a ser desconocido y se suele suponer que $\mathrm{p}=\mathrm{q}=0.5$ que es la opción más segura.

q: es la proporción de individuos que no poseen esa característica, es decir, es 1-p.

n: es el tamaño de la muestra (número de encuestas que se ejecutarán). (Networks, 2001 2013). 
Ecuación aplicada que sirvió para determinar el número de familias que debían ser encuestadas, cuya muestra será considerada estadísticamente como válida:

$$
\begin{gathered}
n=\frac{(1.65)^{2} *(0.5) *(1-0.5) *(3481)}{0.1^{2}(3481-1)+1.65^{2} *(0.5) *(1-0.5)} \\
n=\frac{2369,255625}{35.480625} \\
n=66.7760=6
\end{gathered}
$$

De acuerdo a la ecuación resultante, se demuestra que la muestra óptima para que los resultados sean válidos y confiables es de 66.67 familias, con las cuales se procede a la aplicación de una encuesta en la que se describirán los diversos espacios de la casa, usos y actividades desarrolladas por integrantes de la familia.

\section{Técnicas e instrumentos}

Se realizó una encuesta administrada por el investigador como técnica de recogida de información con la cual a través de preguntas cerradas y abiertas se pretendía conocer la realidad individual de los integrantes de la familia desde las actividades que realiza, espacios de mayor uso, horarios, estatura, peso, etc.

\section{Procedimiento}

Se parte de la aplicación de la ecuación de muestreo estratificado para obtener el valor de la muestra $(\mathrm{N})$ la cual tendrá un nivel confiabilidad del 90\%, resultado de ésta se determina que $\mathrm{N}=67$. Una vez obtenido éste valor se aplican encuestas a las 67 familias, considerando a cada uno de los integrantes como autores principales para proporcionar su información; los resultados exponen que la casa promedio del cantón Girón cuenta con: cocina, comedor, sala, estudio y 4 dormitorios. Por temas explicativos y aplicativos se ejemplifica la aplicación de la ecuación de equilibrio térmico con una familia escogida al azar, a la cual nombraremos CQ, conformada por: padre, madre, hijo y tío; en base a la información proporcionada en la encuesta por cada uno de ellos, se logra aplicar la ecuación de equilibrio térmico de manera individual para la cocina, sala, comedor, estudio y dormitorio; al realizar éste proceso en el que se utiliza la ecuación mencionada se demuestra la eficacia y utilidad de la misma para obtener un confort higro-térmico en los diferentes espacios de la vivienda.

Para aplicar la ecuación de equilibrio térmico se realiza el siguiente procedimiento, en éste caso se utilizará como referencia los datos obtenidos en la encuesta a quién ejerce el rol de padre de la familia CQ

\section{Metabolismo}

Espacio de vivienda: cocina 
Actividad: lavar los platos

Valor de metabolismo en la cocina: 134W, 249W

Superficie corporal: $1,9965 \mathrm{~m}^{2}$

$\mathrm{M}=443,12 \mathrm{~W}$

\section{Radiación}

$$
R=e^{*} \delta^{*} A * \Delta T
$$

Ecuación 3.

$\mathrm{R}=(0,75)^{*}\left(5,6703 * 10-8 \mathrm{watt} / \mathrm{m}^{2} \mathrm{k}^{4}\right) *\left(1,9965 \mathrm{~m}^{2}\right)^{*}\left(296,05^{0} \mathrm{~K}\right)-\left(292,30^{0} \mathrm{~K}\right)^{4}$

$\mathrm{R}=(0,75) *\left(5,6703 * 10-8 \mathrm{watt} / \mathrm{m}^{2} \mathrm{k}^{4}\right) *\left(1,9965 \mathrm{~m}^{2}\right) *\left(3,75^{0} \mathrm{~K}^{4)}\right.$

$\mathrm{R}=3,18 \times 10^{-7} \mathrm{~W}$ (este resultado implica que la persona esta desnuda, como no es el caso, $\mathrm{R}=7,95 \times 10^{-8} \mathrm{~W}$.

\section{Conducción}

$$
\mathbf{C d}=\mathbf{A} * \mathbf{C t} * \Delta \mathbf{T}
$$

Ecuación 8.

Vestimenta: medias, camiseta manga corta, pantalones normales, zapatos suela fina.

Resistividad total: $0,074 \mathrm{~m}^{2}$ gradoC/W

Conductancia:

$$
\text { Resistividad }
$$

Conductancia total: $13,51 \mathrm{~W} / \mathrm{m}^{2}$ gradoC $+(0,34 / 0,01) \mathrm{W} / \mathrm{m}^{2}$ gradoC

Conductancia total: $47,51 \mathrm{~W} / \mathrm{m}^{2}$ gradoC

$\mathrm{Cd}:(1,9965 \mathrm{~m} 2) *\left(47,51 \mathrm{~W} / \mathrm{m}^{2} \operatorname{gradoC}\right) *\left(3,74^{0} \mathrm{C}\right)$

Cd: $354,75 \mathrm{~W}$

\section{Convección}

$$
\mathrm{Cv}=\mathrm{hc} * \mathrm{~A} * \Delta \mathrm{T}
$$

Ecuación 6.

$\mathrm{Cv}:\left(10 \mathrm{~W} / \mathrm{m} 2^{0} \mathrm{C}\right) *(1,9965 \mathrm{~m} 2) *\left(3,74^{0} \mathrm{C}\right)$

$\mathrm{Cv}: 74,67 \mathrm{~W}$

\section{Evaporación}

$$
\mathrm{Qe}=666,66 * \mathrm{e}^{*} \mathrm{v}
$$

Ecuación 9.

Qe:666,66hora/Kg*1,5Kg/h

Qe:999,999

$$
\mathrm{M}+/-\mathbf{R}+/-\mathbf{C d}+/-\mathbf{C v}-\mathbf{E}=\mathbf{0}
$$

Ecuación 1.

$443,12 \mathrm{~W}+7,95 \times 10^{-8} \mathrm{~W}-354,75 \mathrm{~W}-47,67 \mathrm{~W}-999,99=0$ 
Como resultado de la ecuación obtenemos $-986,289$ al ser un resultado negativo concluimos que el individuo está enfriándose, se reduce la circulación sanguínea hacia la piel.

Este procedimiento se ejecuta en cada uno de los espacios de la vivienda y por los cuatro integrantes de la familia CQ cuyo ejemplo se está utilizando para la ejemplificación.

\section{Resultados.}

Luego de ejecutado el proceso de investigación y de aplicar la ecuación de equilibrio térmico para cada uno de los integrantes de la familia en los cinco espacios de la vivienda: cocina, dormitorio, sala, comedor y estudio, y contrastando con los resultados de la encuesta realizada por los mismos, se puede obtener las siguientes tablas (tabla 7 y Figura 1.), mismas en las que se visualizará el valor de balance térmico y las recomendaciones generales para lograr que los espacios de uso frecuente por los integrantes de la familia puedan brindar ese confort higro-térmico anhelado, con lo cual también se puede elaborar un prototipo de vivienda unifamiliar con un adecuado confort-higrotérmico realizando dicho proceso.

Tabla 7. Cálculo del balance térmico en los diferentes espacios de la vivienda para los 4 integrantes

\begin{tabular}{|c|c|c|c|c|c|}
\hline INTEGRANTE & DORMITORIO & SALA & COMEDOR & COCINA & ESTUDIO \\
\hline \multirow{8}{*}{ Papá } & $\mathrm{R}=\mathrm{e}^{*} \sigma^{*} \mathrm{~A}^{*} \Delta \mathrm{T}$ & $\mathrm{R}=\mathrm{e}^{*} \boldsymbol{\sigma} * \mathrm{~A} * \Delta$ & $\mathrm{R}=\mathrm{e}^{*} \sigma^{*} \mathrm{~A}^{*} \Delta \mathrm{T}$ & $R=e^{*} \sigma^{*} A^{*} \Delta T$ & $R=e^{*} \sigma^{*} A^{*} \Delta T$ \\
\hline & $\mathrm{R}=4,11449 \mathrm{E}-06$ & & $R=4,11449 E-06$ & $\mathrm{R}=7,95 \times 10^{-8}$ & $R=4,11449 E-06$ \\
\hline & $\mathrm{Cd}=\mathrm{A} * \mathrm{Ct}^{*} \Delta \mathrm{T}$ & $C d=A * C t * \Delta T$ & $\mathrm{Cd}=\mathrm{A} * \mathrm{Ct} * \Delta \mathrm{T}$ & $C d=A * C t * \Delta T$ & $C d=A * C t * \Delta T$ \\
\hline & $\begin{array}{l}\mathrm{Cd}= \\
350,6944667\end{array}$ & $\mathrm{Cd}=\quad-$ & $\begin{array}{l}\mathrm{Cd}=- \\
350,6944667\end{array}$ & $C d=-354,75$ & $\begin{array}{l}\mathrm{Cd}= \\
350,6944667\end{array}$ \\
\hline & $C v=h c * A * \Delta T$ & $C v=h c * A * A T$ & $C v=h c^{*} A * \Delta T$ & $C y=-7467$ & $C v=h c * A * \Delta T$ \\
\hline & $\mathrm{Cv}=$ & & $\mathrm{Cv}=$ & & $C v=$ \\
\hline & 197,1449492 & $\begin{array}{l}C v= \\
197,1449492\end{array}$ & 197,1449492 & $\begin{array}{l}Q e=666,66 * e v \\
Q e=-999,99\end{array}$ & 197,1449492 \\
\hline & $\begin{array}{l}\mathrm{Qe}=666,66 * \mathrm{ev} \\
\mathrm{Qe}=-10,5\end{array}$ & $\begin{array}{l}Q e=666,66 * e v \\
Q e=-10,5\end{array}$ & $\begin{array}{l}Q e=666,66 * \text { ev } \\
Q e=-10,5\end{array}$ & & $\begin{array}{l}\mathrm{Qe}=\mathbf{6 6 6 , 6 6} * \mathrm{ev} \\
\mathrm{Qe}=-10,5\end{array}$ \\
\hline \multirow{7}{*}{ Mamá } & $R=e^{*} \sigma{ }^{*} A * \Delta T$ & $\begin{array}{l}\mathrm{R}=\mathrm{e}^{*} \boldsymbol{\sigma}{ }^{*} \mathrm{~A} * \Delta \\
\mathrm{T}\end{array}$ & $R=e^{*} \sigma^{*} A * \Delta T$ & $R=e^{*} \sigma^{*} A^{*} \Delta T$ & $R=e^{*} \sigma^{*} A^{*} \Delta T$ \\
\hline & $R=3,68011 \mathrm{E}-07$ & $\mathrm{R}=3.68011 \mathrm{E}-07$ & $R=3,68011 E-07$ & $\mathrm{R}=3,68011 \mathrm{E}-07$ & $R=3,68011 E-07$ \\
\hline & $C d=A * C t * \Delta T$ & $C d=A * C t * \Delta T$ & $\mathrm{Cd}=\mathrm{A} * \mathrm{Ct} * \Delta \mathrm{T}$ & $C d=A * C t * \Delta T$ & $C d=A * C t * \Delta T$ \\
\hline & $\begin{array}{l}C d=- \\
31,36706668\end{array}$ & $\begin{array}{l}\mathrm{Cd}= \\
31,36706668\end{array}$ & $\begin{array}{l}\mathrm{Cd}= \\
31,36706668\end{array}$ & $\begin{array}{l}\mathrm{Cd}= \\
31,36706668\end{array}$ & $\begin{array}{l}\mathrm{Cd}= \\
31,36706668\end{array}$ \\
\hline & $C v=h c^{*} A * \Delta T$ & $C v=h c * A * \Delta T$ & $C v=h c^{*} A * \Delta T$ & $C v=h c^{*} A * \Delta T$ & $C v=h c * A * \Delta T$ \\
\hline & $\begin{array}{l}C v= \\
17,63318031\end{array}$ & $\begin{array}{l}C v= \\
17,63318031\end{array}$ & $\begin{array}{l}C v= \\
17,63318031\end{array}$ & $\begin{array}{l}\mathrm{CV}= \\
17,63318031\end{array}$ & $\begin{array}{l}\mathrm{Cv}= \\
17,63318031\end{array}$ \\
\hline & $\mathrm{Qe}=666,66^{*} \mathrm{ev}$ & $\mathrm{Qe}=666,66^{*} \mathrm{ev}$ & $\mathrm{Qe}=666,66{ }^{*} \mathrm{ev}$ & $\mathrm{Qe}=666,66{ }^{*} \mathrm{ev}$ & $\mathrm{Qe}=666,66^{*} \mathrm{ev}$ \\
\hline
\end{tabular}


ISSN: 2602-8085

Vol. 5, N², p. 149-164, Abril-Junio, 2021

\begin{tabular}{|c|c|c|c|c|c|}
\hline & $\mathrm{Qe}=-10,5$ & $\mathrm{Qe}=-10,5$ & $\mathrm{Qe}=-10,5$ & $\mathrm{Qe}=-10,5$ & $\mathrm{Qe}=-10,5$ \\
\hline \multirow{7}{*}{ Hijo } & $R=e^{*} \sigma^{*} A * \Delta T$ & $\begin{array}{l}\mathrm{R}=\mathrm{e}^{*} \mathrm{\sigma}^{*} \mathrm{~A} * \Delta \\
\mathrm{T}\end{array}$ & $R=e^{*} \sigma^{*} A^{*} \Delta T$ & $R=e^{*} \sigma^{*} A * \Delta T$ & $R=e^{*} \sigma^{*} A^{*} \Delta T$ \\
\hline & $R=3,92882 E-07$ & \multirow{2}{*}{$\begin{array}{l}\mathrm{R}=3,92882 \mathrm{E}-07 \\
\mathbf{C d}=\mathbf{A} * \mathbf{C t} * \boldsymbol{\Delta} \mathbf{T}\end{array}$} & $R=3,92882 E-07$ & $R=3,92882 E-07$ & $R=3,92882 E-07$ \\
\hline & $C d=A * C t * \Delta T$ & & $C d=A * C t * \Delta T$ & $C d=A * C t * \Delta T$ & $\mathrm{Cd}=\mathrm{A} * \mathrm{Ct}{ }^{*} \Delta \mathrm{T}$ \\
\hline & $\begin{array}{l}C d=- \\
33,48692777\end{array}$ & \multirow{2}{*}{$\begin{array}{l}\mathrm{Cd}= \\
33,48692777 \\
\mathbf{C v}=\mathbf{h c} * \mathbf{A} * \boldsymbol{\Delta} \mathbf{T}\end{array}$} & $\begin{array}{l}C d=- \\
33,48692777\end{array}$ & $\begin{array}{l}C d= \\
33,48692777\end{array}$ & $\begin{array}{l}\mathrm{Cd}= \\
33,48692777\end{array}$ \\
\hline & $\mathbf{C v}=h c{ }^{*} A * \Delta T$ & & $C v=h c{ }^{*} A * \Delta T$ & $C v=h c{ }^{*} A * \Delta T$ & $C v=h c * A * \Delta T$ \\
\hline & $\begin{array}{l}C v= \\
18,82487264\end{array}$ & $\begin{array}{l}C v= \\
18,82487264\end{array}$ & $\begin{array}{l}C v= \\
18,82487264\end{array}$ & $\begin{array}{l}C v= \\
18,82487264\end{array}$ & $\begin{array}{l}C V= \\
18,82487264\end{array}$ \\
\hline & $\begin{array}{l}\mathrm{Qe}=666,66{ }^{*} \mathrm{ev} \\
\mathrm{Qe}=-10,5\end{array}$ & $\begin{array}{l}Q e=666,66 * e v \\
Q e=-10,5\end{array}$ & $\begin{array}{l}Q e=666,66 * \text { ev } \\
Q e=-10,5\end{array}$ & $\begin{array}{l}Q e=666,66 * e v \\
Q e=-999,99\end{array}$ & $\begin{array}{l}Q e=666,66 * e v \\
Q e=-10,5\end{array}$ \\
\hline \multirow{5}{*}{ Tío } & $R=e^{*} \sigma^{*} A * \Delta T$ & $\underset{T}{\mathrm{R}=e^{*}} \sigma^{*} A^{*} \Delta$ & $R=e^{*} \sigma^{*} A^{*} \Delta T$ & $R=e^{*} \sigma^{*} A * \Delta T$ & $R=e^{*} \sigma^{*} A^{*} \Delta T$ \\
\hline & $\begin{array}{l}\mathrm{R}=4,11449 \mathrm{E}-06 \\
\mathbf{C d}=\mathbf{A} * \mathbf{C t} * \boldsymbol{\Delta} \mathbf{T}\end{array}$ & $\begin{array}{l}\mathrm{R}=4,11449 \mathrm{E}-06 \\
\mathbf{C d}=\mathbf{A} * \mathbf{C t} * \boldsymbol{\Delta} \mathbf{T}\end{array}$ & $\begin{array}{l}\mathrm{R}=4,11449 \mathrm{E}-06 \\
\mathbf{C d}=\mathbf{A} * \mathbf{C t} * \boldsymbol{\Delta} \mathbf{T}\end{array}$ & $\begin{array}{l}\mathrm{R}=1,67 \times 10^{-5} \\
\mathbf{C d}=\mathbf{A} * \mathbf{C t} * \boldsymbol{\Delta} \mathbf{T}\end{array}$ & $\begin{array}{l}\mathrm{R}=4,11449 \mathrm{E}-06 \\
\mathbf{C d}=\mathbf{A} * \mathbf{C t}^{*} \boldsymbol{\Delta} \mathbf{T}\end{array}$ \\
\hline & $\begin{array}{l}\text { Cd=- } \\
350,6944667 \\
\text { Cv=hc *A* } \Delta T\end{array}$ & $\begin{array}{l}\mathrm{Cd}=- \\
350,6944667 \\
\mathbf{C v}=\mathbf{h c}{ }^{*} \mathbf{A} * \boldsymbol{\Delta} \mathbf{T}\end{array}$ & $\begin{array}{l}\text { Cd }=- \\
350,6944667 \\
\text { Cv=hc * A * } \Delta \mathbf{T}\end{array}$ & $\begin{array}{l}C d=-354,75 \\
\mathbf{C v}=\mathbf{h c} *{ }^{*} * \boldsymbol{\Delta} \mathbf{T} \\
C v=-74,67\end{array}$ & $\begin{array}{l}\mathrm{Cd}= \\
350,6944667 \\
\mathbf{C v}=\mathbf{h c}{ }^{*} \mathbf{A} * \boldsymbol{\Delta} \mathbf{T}\end{array}$ \\
\hline & $\begin{array}{l}C v= \\
197,1449492\end{array}$ & $\begin{array}{l}C v=- \\
197,1449492\end{array}$ & $\begin{array}{l}C v= \\
197,1449492\end{array}$ & $\begin{array}{l}Q e=666,66 * e v \\
Q e=-999,99\end{array}$ & $\begin{array}{l}C v= \\
197,1449492\end{array}$ \\
\hline & $\begin{array}{l}Q \mathbf{Q}=666,66 * \mathrm{ev} \\
\mathrm{Qe}=-10,5\end{array}$ & $\begin{array}{l}Q e=666,66 * e v \\
Q e=-10,5\end{array}$ & $\begin{array}{l}Q e=666,66 * e v \\
Q e=-10,5\end{array}$ & & $\begin{array}{l}Q e=666,66 * e v \\
Q e=-10,5\end{array}$ \\
\hline
\end{tabular}

Fuente: Murillo, 2011.

Elaborado: Cristian Guzmán

\begin{tabular}{|c|c|c|c|c|c|c|c|c|c|c|c|c|}
\hline Usuario & $\begin{array}{l}\text { Edad } \\
\text { años }\end{array}$ & $\begin{array}{c}\text { Peso } \\
\mathrm{Kg}\end{array}$ & $\begin{array}{c}\text { Talla } \\
\mathrm{m}\end{array}$ & Piel & $\underset{\text { iento }}{\text { Arropam }}$ & Actividad & $\begin{array}{c}\text { Ambiente } \\
\text { más } \\
\text { utilizado }\end{array}$ & $\underset{\text { uso }}{\text { Horario de }}$ & $\begin{array}{l}\text { Mes de } \\
\text { análisis más } \\
\text { critico }\end{array}$ & $\begin{array}{l}\text { Perdidas por } \\
\text { evaporación }\end{array}$ & $\begin{array}{c}\text { Equilibrio } \\
\text { Térmico }\end{array}$ & $\begin{array}{l}\text { Estrategias } \\
\text { Generales }\end{array}$ \\
\hline \multirow[t]{2}{*}{ Papa } & \multirow[t]{2}{*}{59} & \multirow[t]{2}{*}{82} & \multirow[t]{2}{*}{1,75} & \multirow[t]{2}{*}{ Tipo III } & \multirow{10}{*}{$\begin{array}{c}\text { Traje con } \\
\text { interior } \\
\text { ligero, } \\
\text { camisa } \\
\text { cortay } \\
\text { pantalon } \\
\text { ligeroo } \\
\text { ropa } \\
\text { similar. }\end{array}$} & \multirow{2}{*}{$\begin{array}{l}\text { Sertado con } \\
\text { movimiento } \\
\text { moderado / Dormir }\end{array}$} & Sala & Noche & \multirow[t]{2}{*}{ Agosto } & \multirow{2}{*}{$\begin{array}{l}\text { Evaporación } \\
\text { por } \\
\text { respiración } \\
10,5 \mathrm{w}\end{array}$} & $-320,75$ & \multirow{2}{*}{$\begin{array}{c}\text { Gamancia de } \\
\text { Calor en } \\
\text { dormitorios y } \\
\text { salas en la noche }\end{array}$} \\
\hline & & & & & & & Donmitorio & Noche & & & $-283,35$ & \\
\hline \multirow{4}{*}{ Mama } & \multirow{4}{*}{60} & \multirow{4}{*}{78} & \multirow{4}{*}{168} & \multirow{4}{*}{ Tipo III } & & \multirow{4}{*}{$\begin{array}{c}\text { Trabajo marual } \\
\text { moderado / Dormir }\end{array}$} & Cocina & $\begin{array}{c}\text { Nañzna } \\
\text { terde noche }\end{array}$ & \multirow{4}{*}{ Agosto } & \multirow{2}{*}{$\begin{array}{c}\text { Tasa de } \\
\text { evaporación } \\
\text { por sudoración } \\
1,5 \mathrm{~kg} / \mathrm{h}\end{array}$} & $-1098,78$ & \multirow{4}{*}{$\begin{array}{l}\text { Ganancia de } \\
\text { Calor en; } \\
\text { Cocina mañana } \\
\text { nocke, y sala } \\
\text { donmitorios en lo } \\
\text { noche }\end{array}$} \\
\hline & & & & & & & Lavanderia & Tarde & & & $-1051,08$ & \\
\hline & & & & & & & Dormitorio & Noche & & \multirow{2}{*}{$\begin{array}{l}\text { Evaporación } \\
\text { por } \\
\text { respiración } \\
10,5 \mathrm{w}\end{array}$} & $-308,95$ & \\
\hline & & & & & & & Sala & Noche & & & $-271,24$ & \\
\hline \multirow[t]{2}{*}{ Hijo } & \multirow[t]{2}{*}{19} & \multirow[t]{2}{*}{68} & \multirow[t]{2}{*}{185} & \multirow[t]{2}{*}{ Tipo III } & & $\begin{array}{l}\text { Sertado con } \\
\text { movimiento }\end{array}$ & Sala & Noche & \multirow{2}{*}{ Agosto } & \multirow{2}{*}{$\begin{array}{l}\text { Evaporación } \\
\text { por } \\
\text { respiración } \\
10,5 \mathrm{w}\end{array}$} & $-258,69$ & \multirow{4}{*}{$\begin{array}{c}\text { Ganarcia de } \\
\text { Calor en } \\
\text { dormitorios y } \\
\text { salas en la noche } \\
\text { Ganarcia de } \\
\text { Calor en } \\
\text { domitorios y } \\
\text { salas en la noche }\end{array}$} \\
\hline & & & & & & moderado / Dormir & Donmitorio & Noche & & & $-303,31$ & \\
\hline & & & & & & & Sala & Noche & \multirow[b]{2}{*}{ Agosto } & \multirow[b]{2}{*}{$\begin{array}{l}\text { Evaporación } \\
\text { por } \\
\text { respiracion } \\
10,5 \mathrm{w}\end{array}$} & $-318,98$ & \\
\hline Tio & 55 & 80 & 175 & Tipo III & & $\begin{array}{l}\text { Sertodo con } \\
\text { movimiento } \\
\text { moderado / Dormir }\end{array}$ & Dormitorio & Noche & & & $-280,10$ & \\
\hline
\end{tabular}

Figura 1. Resultados de confort higro-térmico.

Fuente: Guzmán, 2016. 


\section{Conclusiones.}

- En el cantón Girón existen viviendas que en su mayoría cuentan con: cocina, sala, comedor, estudio y cuatro dormitorios pero que no han sido diseñadas con un criterio enfocado al confort higrotérmico, lo que genera un malestar en las personas que habitan la unidad de vivienda. Al utilizar la fórmula del balance o equilibrio térmico podemos conocer de manera más clara y precisa cuales son las condiciones que requieren los diferentes espacios para que puedan sus habitantes percibir o sentirse en confort, sin embargo, por el tiempo que esto requiere y los costos que implican no han sido adaptadas por la ciudadanía llegando a convertirse en un círculo vicioso en el que el malestar y problema están latentes, aunque la solución esté cercana.

- Las personas experimentan diferentes sensaciones térmicas corporales de acuerdo a la actividad que se encuentre realizando en un determinado espacio, esta variación depende exclusivamente del balance higrotérmico y la vestimenta que son directamente proporcionales a la actividad que realiza, por cuanto al aplicar la fórmula se abre un abanico de posibilidades para plantear recomendaciones que permitan alcanzar los niveles de confort anhelados que pueden ir desde ampliar los espacios físicos, considerar diferentes materiales en la construcción como adobe, ladrillo, etc., colocar espacios de ingreso a la luz por ende al calor, todo esto considerando que el metabolismo arrojará siempre un resultado positivo puesto que es la ganancia del calor generada por el cuerpo, la radiación es positiva debido a que la radicación del sol es directa, la conducción es negativa porque el cuerpo humano es expuesto al contacto con el frío, la convección resulta negativa debido a que la temperatura del aire es menor a la temperatura interna del cuerpo humano, y la evaporación siempre arroja un valor negativo debido a la evapotranspiración; cuando el resultado final de la ecuación nos arroja un resultado negativo se entiende que el cuerpo está enfriándose y por el contrario si el resultado es positivo el cuerpo se está calentando.

\section{Agradecimiento.}

El presente artículo es parte del trabajo de investigación y titulación del Programa de Maestría en Construcción con Mención en Administración de la Construcción Sustentable de la Universidad Católica de Cuenca, por ello agradecemos a todos y cada uno de los instructores por los conocimientos e información brindados para la elaboración del trabajo.

\section{Referencias bibliográficas.}

Cancino, C., Cortés, A., (2015). Confort higro-térmico en vivienda social y la percepción del habitante. Revista INVI, 30 (85). Recuperado desde: h https://scielo.conicyt.cl/scielo.php?script=sci_arttext\&pid=S0718$83582015000300008 \&$ lang=es 
Czajkowski, J., Diulio, M., García, M., Marcilese, M., Salvetti, B., Vagge, C. (2012). E Evaluación del confort higrotérmico invernal en viviendas unifamiliares del Gran la Plata mediante auditorias. Avances en energía renovable y medio ambiente, 16. $\mathrm{R}$ Recuperado desde: http://sedici.unlp.edu.ar/bitstream/handle/10915/89568/Documento_completo.pd f- $\quad$ PDFA.pdf?sequence $=1 \&$ isAllowed $=y$

Garduño, A., Hernández, S. (2010). Tecnologías actuales aplicadas al desarrollo urbano s sustentable. Acta Universitaria, 20(1),25-34. Recuperado desde: https://www.redalyc.org/articulo.oa?id=416/41613084003

Guzmán, C.(2016). Recomendaciones bioclimáticas para el diseño de vivienda unifamiliar en un clima ecuatorial mesotérmico semihúmedo ubicado en el cantón Girón de la Provincia del Azuay. Repositorio UCACUE.

Lisandro, S. (2015). Los intelectuales y el poder en la producción del hábitat residencial de interés social. Revista INVI, 30(85),9-20. Recuperado desde: https://www.redalyc.org/articulo.oa?id=258/25842618001

Murillo, R. (2011). Arquitectura bioclimática. Guayaquil: Editorial de publicaciones de la Universidad Católica de Santiago de Guayaquil.

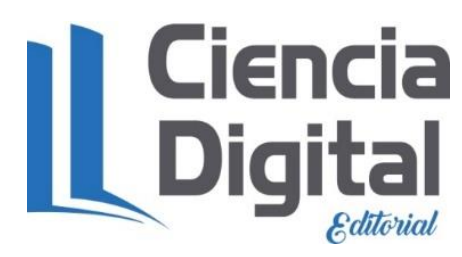




\section{PARA CITAR EL ARTÍCULO INDEXADO.}

Guzmán Clavijo, C. R., \& Alvear Calle, D. A. (2021). Aplicación de la Ecuación del Balance Térmico para determinar el confort higrotérmico en la vivienda unifamiliar en el

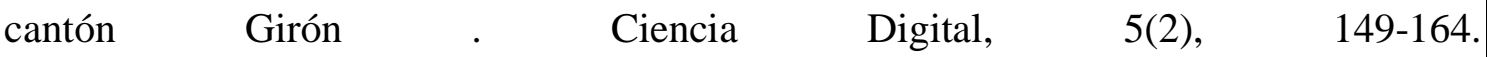
https://doi.org/10.33262/cienciadigital.v5i2.1722

\section{Ciencia \\ LDigital}

El artículo que se publica es de exclusiva responsabilidad de los autores y no necesariamente reflejan el pensamiento de la Revista Ciencia Digital.

El artículo queda en propiedad de la revista y, por tanto, su publicación parcial y/o total en otro medio tiene que ser autorizado por el director de la Revista Ciencia Digital.
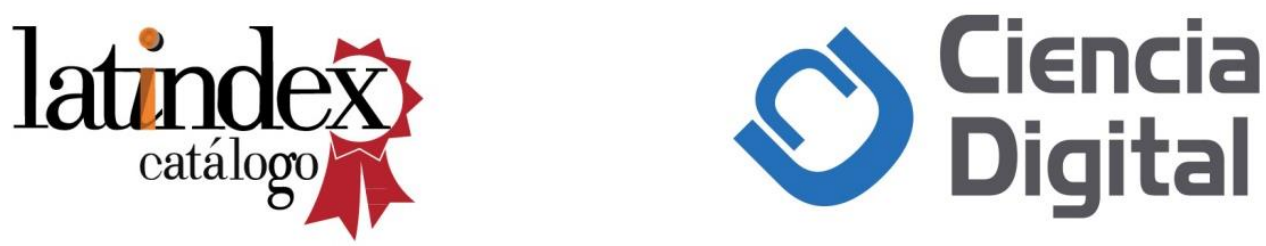